\title{
Wound Infiltration with Levobupivacaine, Ketorolac, and Adrenaline for Postoperative Pain Control after Spinal Fusion Surgery
}

\author{
Valerio Pace ${ }^{1}$, Arif Gul ${ }^{2}$, Varadarajan Prakash ${ }^{2}$, Chang Park $^{1}$, Giacomo Placella ${ }^{3}$, Geoffrey Raine ${ }^{4}$ \\ ${ }^{1}$ Department of Trauma and Orthopaedic Surgery, The Royal National Orthopaedic Hospital, Stanmore, UK \\ ${ }^{2}$ Department of Trauma and Orthopaedics, The Princess Alexandra Hospital NHS Trust, Harlow, UK \\ ${ }^{3}$ Department of Trauma and Orthopaedics, IRCCS San Raffaele Hospital, Milan, Italy \\ ${ }^{4}$ Department of Anaesthetics, The Princess Alexandra Hospital NHS Trust, Harlow, UK
}

Study Design: This study enrolled patients in from a single center who underwent primary spinal fusion procedure and divided them into two groups (group-control study).

Purpose: Good local infiltration can reduce postoperative analgesic requirements and enable expedited discharge. Administration of a combination of levobupivacaine ( $200 \mathrm{mg} / 100 \mathrm{~mL}, 0.9 \%$ normal saline), ketorolac $(30 \mathrm{mg})$, and adrenaline $(0.5 \mathrm{mg})$ as a wound infiltrate is recommended at an optimum combination.

Overview of Literature: There is currently no consensus on the optimum intraoperative local infiltration of spinal surgery patients undergoing operative fusion.

Methods: Patients who were enrolled in two spinal centers (over 24 months) undergoing primary spinal fusion procedures were allocated into two groups, comparing the type of local infiltration used at the time of the procedure. Group 1 received the combination of levobupivacaine $(200 \mathrm{mg})$, ketorolac $(30 \mathrm{mg})$, and adrenaline $(0.5 \mathrm{mg})$, while group 2 received other types of local anesthetics. Primary outcome measures include patient-controlled analgesia (PCA) use, morphine consumption, and length of hospital stay. Secondary outcome measure are as follows: days of physiotherapy, pain score, side effects, and complications.

Results: There are a total of 140 patients enrolled. Seventy-five patients enrolled were allocated to group 1, receiving the study combination, and 65 patients were assigned in group 2, receiving other local infiltrations. All primary outcome measures (consumption of morphine, use of PCA, and length of stay) were significantly higher in group 2 than the study combination $(p<0.05)$. The secondary outcomes of pain scores and days of physiotherapy values were also significantly higher in group $2(p<0.05)$. Patient satisfaction questionnaires gave significantly better results in group $1(p<0.05)$. There were no significant statistical differences with regard to any postoperative complications between the two groups.

Conclusions: Our data suggest that the studied wound infiltration is a safe and feasible option that could provide good postoperative pain control without significant side effects. It also allowed to reduce dependence of opioids and PCA, earlier postoperative mobilization, lower pain scores postoperatively, and reduced hospital stay.

Keywords: Wound infiltration; Spinal surgery; Spinal fusion; Pain control; Postoperative management; Pain scores

Received Mar 14, 2020; Revised Apr 6, 2020; Accepted Apr 9, 2020

Corresponding authors: Valerio Pace

Department of Trauma and Orthopaedic Surgery, The Royal National Orthopaedic Hospital, Brockley Hill, Stanmore, Middlesex, HA7 $4 \mathrm{LP}, \mathrm{UK}$

Tel: +44-020-3947-0100, Fax: +44-20-8909-5349, E-mail: valeriopace@doctors.org.uk 


\section{Introduction}

Currently, there is no clear consensus on the optimum combination of local wound infiltration analgesics for postoperative pain control in spinal surgery. Several paraspinal musculature infiltration mixes during operative procedure have been investigated for efficacy with conflicting results $[1,2]$.

Postoperative pain following spinal surgery can lead to a series of significant complications, including delayed mobilization and increased use of opioids postoperatively. These postoperative challenges in patients who have undergone spinal surgery can make postoperative rehabilitation more challenging, resulting in requiring more physiotherapy and increasing the length of hospital stay.

Current postoperative analgesic regimens are in accordance with the World Health Organization (WHO)'s pathway regarding the use of opioids and patient-controlled analgesia (PCA) [3]. Opiates provide excellent analgesic effect, and its side effects are well documented [4]. There have been studies comparing the efficacy of postoperative analgesia, that is, comparing continuous epidural analgesia with PCA, along with the use of transdermal patches and methods to reduce traditional opioid use postoperatively by supplementing the pain regimen with other agents, such as ketamine, ketorolac, and GABA (gammaaminobutyric acid) receptor antagonists [5-14].

This paper is designed to investigate the role of paraspinal wound infiltration in postoperative pain control. By optimizing the local infiltration regimen at the time of procedure, one would aim to reduce the requirements of postoperative systemic analgesia with its more significant side effect profile.

We hypothesize the effectivity of administering a combination of levobupivacaine $(200 \mathrm{mg} / 100 \mathrm{~mL}$ in $0.9 \%$ normal saline [ $\mathrm{N}$-saline]), ketorolac $(30 \mathrm{mg})$, and adrenaline $(0.5 \mathrm{mg})$ as a wound infiltrate at the end of spinal fusion surgery and a regimen that could reduce the requirement of postoperative analgesia, including opioids and PCA, and in turn shorten the length of stay and postoperative mobilization as compared to current regimens.

\section{Materials and Methods}

All patients undergoing elective spinal fusion surgery were identified at two spinal units over a 24 -month period. This included fusions at the cervical, thoracic, and lumbar levels (single- or two-level fusions). The procedures were performed by the same team.

Patients were randomly allocated into one of two groups. Group 1 received the wound infiltration intervention of a combination of levobupivacaine $(200 \mathrm{mg} / 100 \mathrm{~mL}$ in $0.9 \% \mathrm{~N}$-saline), ketorolac (30 mg), and adrenaline $(0.5$ $\mathrm{mg})$. Group 2 were the control patients who received the regular wound infiltration of the surgeon and anesthetists' discretion.

All patients followed the standard postoperative pain management protocol in accordance with the WHO pain ladder pathway [3]. The primary outcome measures of the study were PCA postoperative use, postoperative morphine consumption, and length of stay in the hospital. Secondary outcome measures included the required days of physiotherapy, 7-day postoperative pain score (on a scale from 0 to 3 as recently set up by the local Trust policies) along with any analgesic side effects and postoperative complications. Patient satisfaction following the procedure was also recorded by means of a postoperative questionnaire.

Patients were prescribed postoperative analgesia according to Trust drug charts, and pain scores were recorded in the Trust observation charts. The intraoperative infiltration regimen was written down in the operation notes, and all the data were retrospectively collected and stored using an electronic spreadsheet. The study was conducted in accordance with the principles of the Declaration of Helsinki and its amendments, and informed consents were given by all included patients

\section{Results}

A total of 140 patients were enrolled in the study and matched our inclusion criteria. Overall, 53 patients were male and 87 were female. The age range was from 31 to 84 years. Seventy-five patients were assigned to group 1, receiving the intervention regimen, and 65 to group 2 as the control. Of the 140 patients, 49 had cervical fusion, 15 had thoracic fusion, and 76 had lumbar fusion.

In group 1, 31 patients were male and 44 were female who received the new combination for local infiltration. The mean age was $48.4 \pm 2.8$ years, and the mean body mass index (BMI) was $27.4 \pm 1.4 \mathrm{~kg} / \mathrm{m}^{2}$. The mean duration of surgery was $96.4 \pm 16.8$ minutes. Moreover, 25 patients had cervical fusion, 10 had thoracic fusion, and 40 had lumbar fusion. 
Table 1. Comparison of results group 1 versus group 2

\begin{tabular}{lcc|}
\hline Variable & Group 1 & Group 2 \\
\hline PCA day $1(\%)$ & 51 & 62 \\
\hline PCA day 2 (\%) & 16 & 22 \\
\hline Morphine overall $(\mathrm{mg})$ & 18.2 & 23.1 \\
\hline Morphine day $1(\mathrm{mg})$ & 18.1 & 20.9 \\
\hline Morphine day 2 $(\mathrm{mg})$ & 22.3 & 24.1 \\
\hline Pain score overall & 1.3 & 1.6 \\
\hline Pain score day 1 & 1.5 & 1.9 \\
\hline Pain score day 2 & 1.6 & 1.8 \\
\hline Days of physiotherapy (day) & 4.5 & 5.6 \\
\hline Length of stay (day) & 6.8 & 7.9 \\
\hline Patient happy (\%) & 85 & 74 \\
\hline No. of complications & 3 & 3 \\
\hline PCA. patient-contoled analgesia. & & \\
\hline
\end{tabular}

PCA, patient-controlled analgesia.

Of the 65 patients in group 2, 22 were male patients and 43 female patients. The mean age was $48.8 \pm 3.1$ years, and the mean BMI was $28.1 \pm 1.6 \mathrm{~kg} / \mathrm{m}^{2}$. The mean duration of surgery was $95.9 \pm 16.4$ minutes. Of patients allocated in group 2, 24 had cervical fusion, five had thoracic fusion, and 36 had lumbar fusion.

Of the primary outcome measures with the intervention regimen, $51 \%$ of patients were still requiring PCA on day one and $16 \%$ until day 2 . In group 2, PCA was used postoperatively by $62 \%$ of patients in day one and $22 \%$ until day 2 (Table 1 ).

The mean daily opioid use of oral morphine in group 1 was $18.2-18.1 \mathrm{mg}$ in day 1 postoperative and $22.3 \mathrm{mg}$ in day 2. Meanwhile, the mean daily opioid use in group 2 was $3.1-20.9 \mathrm{mg}$ in day 1 postoperative and $24.1 \mathrm{mg}$ in day 2. For group 1, the mean pain score reported was $1.3-1.5$ on day 1 postoperative and 1.6 the following day, and for group 2 was 1.6-1.9 in day one postoperative and 1.8 in day 2. The average length of stay was 6.8 days for those in group 1 compared to 7.9 days for those in group 2 .

In terms of secondary outcome measures, there were no direct side effects noted from the local wound infiltration in group 1. However, three patients had surgical complications related to the procedure performed who needed to be taken back to the theater for further management. The first patient had an infective case which needed fasciectomy, wash out, and long-term antibiotics. The second one was a case of screw displacement following spinal fusion with impaired postoperative neurology, and the pa- tient was taken back to the theater for urgent metalwork revision, which was successfully performed and returned to normal neurology. One patient developed a superficial wound infection which required 1 week of oral antibiotics with consequent complete resolution. There were no direct side effects noted from patients in group 2 from the local wound infiltration, but there were two cases of postoperative wound infections successfully treated with a week of oral antibiotics.

Patients in group 1, on average, required 4.5 days of physiotherapy, and $85 \%$ of those patients were reported to be happy of the procedure. For those in group 2, the average duration of physiotherapy required was 5.6 days, and $74 \%$ of patients in group 2 reported to be happy with the results of the procedure (Table 1 ).

On statistical analysis, all primary outcome measures covering the consumption of morphine and the use of PCA and length of stay were significantly higher in group 2 than that of group $1(p<0.05)$. The secondary outcomes of pain scores and days of physiotherapy values were also significantly higher in group $2(p<0.05)$. Patient satisfaction questionnaires gave significantly better results in group $1(p<0.05)$. There were no significant statistical differences with regard to any postoperative complications between the two groups.

\section{Discussion}

Peri- and postoperative pain control is one of the factors influencing clinical outcomes for spinal fusion surgery patients as well as patients' overall experience during hospital stay and postoperative period. Poor pain control has been linked with decreased pain satisfaction and increased economic burden due to increased dependence of analgesia and increased length of hospital stay which are important considerations given the increasing cost of healthcare in many nations. Unfortunately, opioids have been reported to have many limitations and side effects despite being the primary treatment of postoperative pain [15]. Therefore, it is imperative that an appropriate analgesic regimen is offered to provide adequate pain relief in order to allow early mobilization and an optimum rehabilitation program postoperatively.

A well-recognized and internationally approved combination of drugs used as wound infiltration at the end of spinal fusion surgery with good results in terms of postoperative pain management and high level of evidence has 
not yet been identified. However, promising but conflicting results have been obtained in several studies.

The synergistic effect of clonidine with bupivacaine, well-established in peripheral nerve blocks, remains controversial in local field block for postoperative analgesia despite resulting in better and prolonged postoperative analgesia in posterior lumbar spine surgeries [16].

Recent studies on pain control in the first postoperative hours following spinal surgery have demonstrated that subarachnoid block results in decreasing the severity of pain but only during the first hours postoperatively. The administration of bupivacaine wound infiltration in patients with lumbar herniated disc seems to avoid high doses of opioids and significant pain relief during the first 2 days postoperatively, allowing rapid rehabilitation [17].

There has been a growing interest in continuous local anesthetic wound infiltration as a non-opioid technique for postoperative pain relief. According to results, the impact of this modality on baseline analgesia after spinal fusion surgery has been inconclusive [10].

Administration of a combination of corticosteroid and bupivacaine in patients undergoing posterior lumbosacral spine surgery had beneficial effects but without statistically significant difference in all subgroup comparisons. However, results showed that the administration of methylprednisolone-bupivacaine provided a favorable effect immediately after posterior lumbosacral spine surgery for discectomy, decompression, and/or spinal fusion without complications [11].

Nonetheless, studies on several paraspinal infiltration mixes for a better pain management in spinal surgery have achieved conflicts in literature [1,2]. Well-powered prospective studies are warranted in the future to determine optimal dosing and confirm benefits of all available measures of pain control following major spinal surgery [15].

Moreover, several studies focusing on different aspects of spinal conditions (including conditions treated with both conservative and surgical management strategies) have shown that an optimum pain management of such patients could allow the best possible rehabilitation, functional outcomes, mobility status, and surgical recovery. This is particularly true for conditions linked to high levels of discomfort and pain, such as idiopathic scoliosis, degenerative scoliosis, and thoracolumbar vertebrae fractures. Patients' safety and experience, functional outcomes, and healthcare costs should always be considered.
These key aspects further confirm that an appropriate pain control is warranted for our cohort of patients which could highlight the importance and relevance of our good results in terms of pain management, healthcare costs, and related safety of the intervention [18-23].

The wound infiltration utilized in this study exhibited good results in terms of postoperative pain control, with a reduced dependence of PCA and opioid analgesia as primary outcome measures. Furthermore, the reduced pain consent to early mobilization and shorter length of stay than that of the control. These factors help reduce postoperative complications and improved patients' experience with pain. Furthermore, all these factors contribute to the reduction of potential healthcare costs for those in group 1 than that of the control. Our combination of levobupivacaine, ketorolac, and adrenaline has not been studied before. To the best of our knowledge, this set of data is the first of its kind available in literature.

\section{Conclusions}

Our data suggest that the examined wound infiltration is a safe and feasible option providing good postoperative pain control without significant side effects. It also allows low usage of opioids, early postoperative mobilization, and short length of hospital stay.

There is paucity of high level of evidence studies in the literature to guide analgesic regimens in patients undergoing spinal fusion surgery. Several mixes and strategies have been used and studied for different cohorts in spinal surgery, and we, the authors, suggest that our combination is a successful intraoperative infiltration that can help reduce dependence on postoperative opioid regimens and improve patient care.

We suggest that a bigger case-control study and a highquality randomized controlled trial to be carried out in order to achieve higher level of evidence and consensus in ways to best set up pain management measures for patients undergoing spinal surgery fusion. In this vision, our study seems to be a good start on the journey to achieve consensus on managing postoperative pain for spinal surgery patients.

\section{Conflict of Interest}

No potential conflict of interest relevant to this article was reported. 


\section{Author Contributions}

Conception and design: VP, AG, VP, CP, GP, GR; administrative support: AG, VP, GR; provision of study materials or patients: VP, AG, GP, GR; collection and assembly of data: VP, AG, VP, CP, GP, GR; data analysis and interpretation: VP, AG, VP, CP, GP, GR; manuscript writing: VP, AG, VP, CP, GP, GR; and final approval of manuscript: VP, AG, VP, CP, GP, GR.

\section{References}

1. Glasser RS, Knego RS, Delashaw JB, Fessler RG. The perioperative use of corticosteroids and bupivacaine in the management of lumbar disc disease. J Neurosurg 1993;78:383-7.

2. Teddy PJ, Fabinyi GC, Kerr JH, Briggs M. Bupivacaine infiltration after lumbar laminectomy: local infiltration in the control of early postoperative lumbar laminectomy pain. Anaesthesia 1981;36:380-3.

3. World Health Organization. Cancer pain relief: with a guide to opioid availability. Geneva: World Health Organization; 1987.

4. Sheffer BW, Kelly DM, Rhodes LN, Sawyer JR. Perioperative pain management in pediatric spine surgery. Orthop Clin North Am 2017;48:481-6.

5. Connolly J 3rd, Javed Z, Raji MA, Chan W, Kuo YF, Baillargeon J. Predictors of long-term opioid use following lumbar fusion surgery. Spine (Phila Pa 1976) 2017;42:1405-11.

6. Toktas ZO, Konakci M, Yilmaz B, et al. Pain control following posterior spine fusion: patient-controlled continuous epidural catheter infusion method yields better post-operative analgesia control compared to intravenous patient controlled analgesia method: a retrospective case series. Eur Spine J 2016;25:160813.

7. Tian P, Fu X, Li ZJ, Ma XL. Comparison of patientcontrolled epidural analgesia and patient-controlled intravenous analgesia after spinal fusion surgery: a meta-analysis of randomized controlled trials. BMC Musculoskelet Disord 2015;16:388.

8. Nielsen RV, Fomsgaard JS, Siegel H, et al. Intraoperative ketamine reduces immediate postoperative opioid consumption after spinal fusion surgery in chronic pain patients with opioid dependency: a randomized, blinded trial. Pain 2017;158:463-70.
9. Kim HJ, Ahn HS, Nam Y, Chang BS, Lee CK, Yeom JS. Comparative study of the efficacy of transdermal buprenorphine patches and prolonged-release tramadol tablets for postoperative pain control after spinal fusion surgery: a prospective, randomized controlled non-inferiority trial. Eur Spine J 2017;26:2961-8.

10. Greze J, Vighetti A, Incagnoli P, et al. Does continuous wound infiltration enhance baseline intravenous multimodal analgesia after posterior spinal fusion surgery?: a randomized, double-blinded, placebocontrolled study. Eur Spine J 2017;26:832-9.

11. Jirarattanaphochai K, Jung S, Thienthong S, Krisanaprakornkit W, Sumananont C. Peridural methylprednisolone and wound infiltration with bupivacaine for postoperative pain control after posterior lumbar spine surgery: a randomized double-blinded placebo-controlled trial. Spine (Phila Pa 1976) 2007;32:609-17.

12. Salem RA, Darweesh EI, Wanis MA, Mohamed AA. Evaluation of the effects of intrathecal bupivacainedexmedetomidine for lumbar spine fusion: a double blinded randomized controlled study. Eur Rev Med Pharmacol Sci 2015;19:4542-8.

13. Elder JB, Hoh DJ, Wang MY. Postoperative continuous paravertebral anesthetic infusion for pain control in lumbar spinal fusion surgery. Spine (Phila Pa 1976) 2008;33:210-8.

14. Quinlan P, Davis J, Fields K, et al. Effects of localized cold therapy on pain in postoperative spinal fusion patients: a randomized control trial. Orthop Nurs 2017;36:344-9.

15. Tomov M, Tou K, Winkel R, et al. Does subcutaneous infiltration of liposomal bupivacaine following single-level transforaminal lumbar interbody fusion surgery improve immediate postoperative pain control? Asian Spine J 2018;12:85-93.

16. Abdel Hay J, Kobaiter-Maarrawi S, Tabet P, et al. Bupivacaine field block with clonidine for postoperative pain control in posterior spine approaches: a randomized double-blind trial. Neurosurgery 2018;82:790-8.

17. Genov PG, Timerbaev VH, Grin AA, Rebrova OY. The choice of perioperative multimodal analgesia in patients with lumbar herniated disc: the preliminary results. Anesteziol Reanimatol 2017;61:214-9.

18. Zaveri A, Pace V, Bhagawati D, Rajamani V, Muthukumar T, Noordeen H. Management of progressive 
late onset scoliosis with magnetic growth rod insertion leading to improvement of neural anomalies: a case report. J Spine Surg 2016;2:324-7.

19. Cervera-Irimia J, Gonzalez-Miranda A, RiquelmeGarcia O, et al. Scoliosis induced by costotransversectomy in minipigs model. Med Glas (Zenica) 2019;16:157-63.

20. Meccariello L, Muzii VF, Falzarano G, et al. Dynamic corset versus three-point brace in the treatment of osteoporotic compression fractures of the thoracic and lumbar spine: a prospective, comparative study. Aging Clin Exp Res 2017;29:443-9.
21. Medici A, Meccariello L, Falzarano G. Non-operative vs. percutaneous stabilization in Magerl's A1 or A2 thoracolumbar spine fracture in adults: is it really advantageous for a good alignment of the spine?: preliminary data from a prospective study. Eur Spine J 2014;23 Suppl 6:677-83.

22. Falzarano G, Rollo G, Bisaccia M, et al. Percutaneous screws CT guided to fix sacroiliac joint in tile C pelvic injury: outcomes at 5 years of follow-up. SICOT J 2018;4:52.

23. Pace V, Bhagawati D, Park C, Molloy S. Delayed presentation of a symptomatic psoas hematoma following lumbar vertebral kyphoplasty for myeloma: a case report. J Orthop Case Rep 2018;8:74-8. 